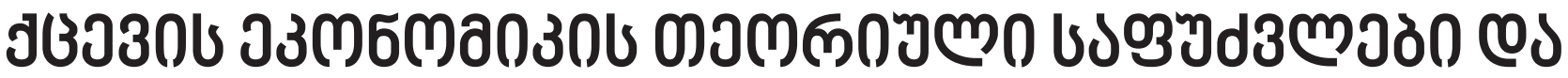

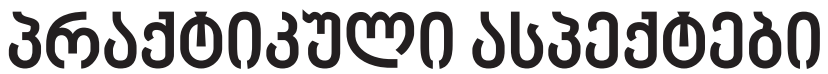

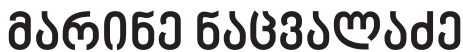

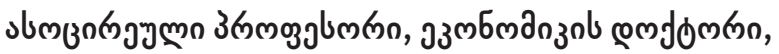

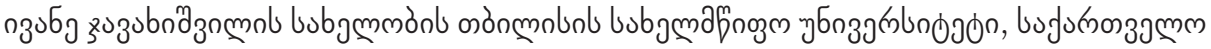
marine.natsvaladze@tsu.ge

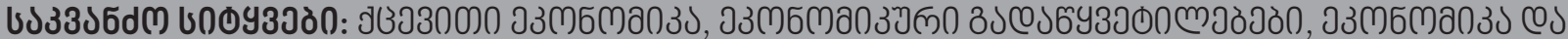

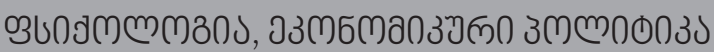

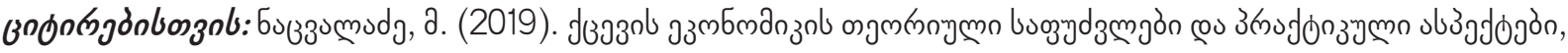

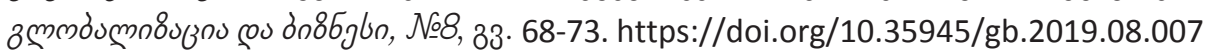

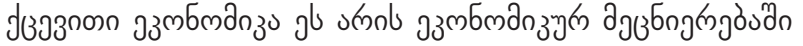

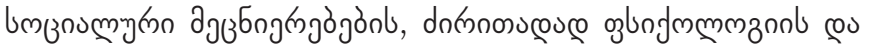

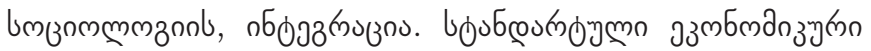

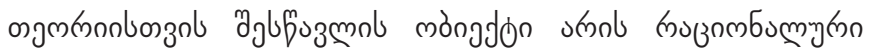

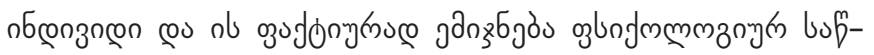

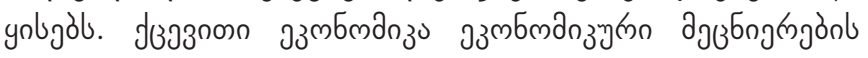

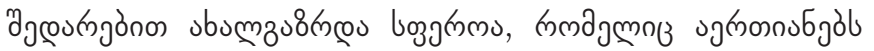

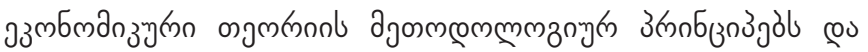

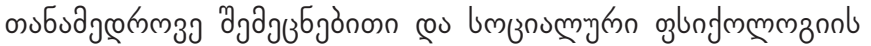

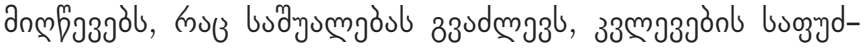

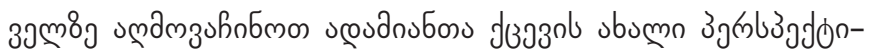

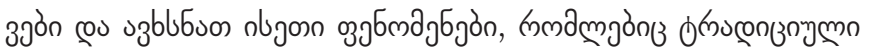
उзलбmanz

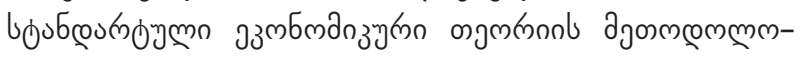

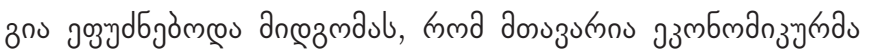

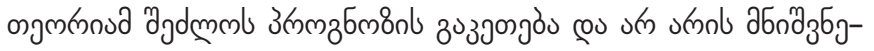

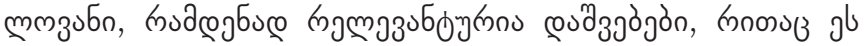

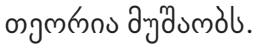

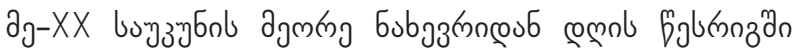

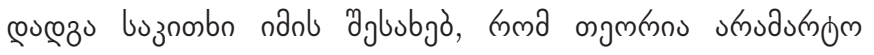

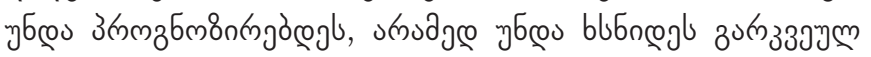

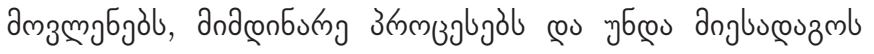

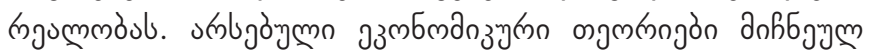

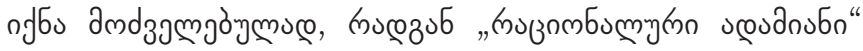

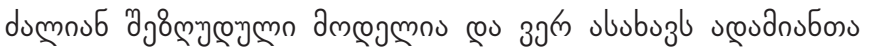

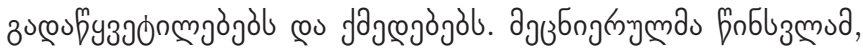

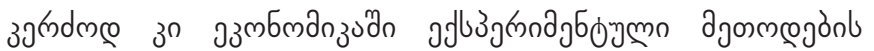

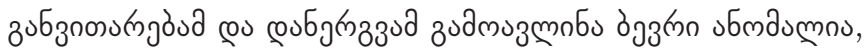

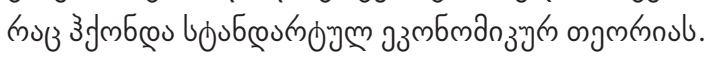

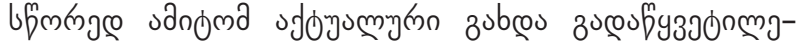

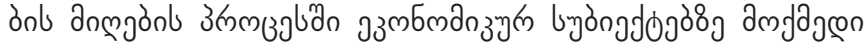

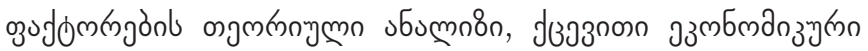

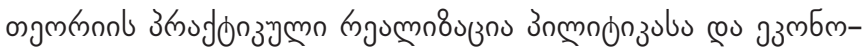
anzo

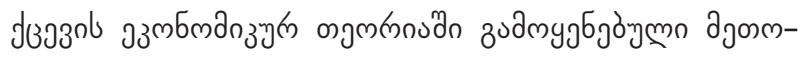

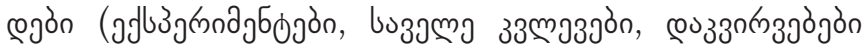

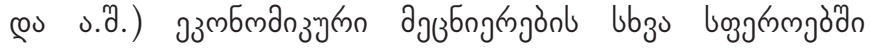

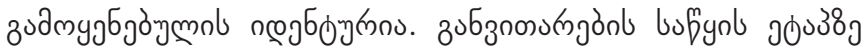

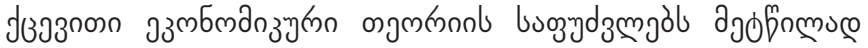

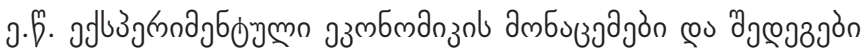
fumamuœ

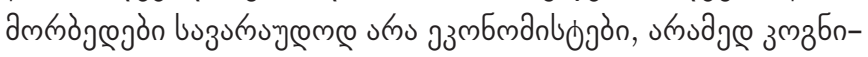

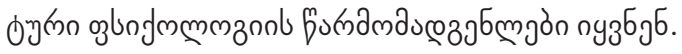

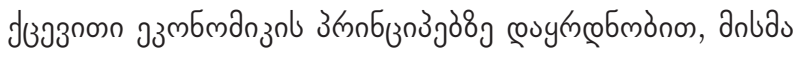

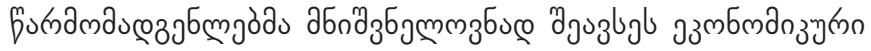

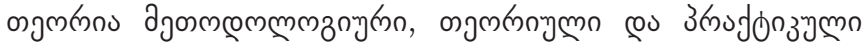

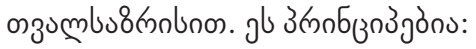

1. эзмбмдаз

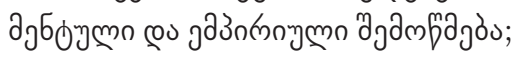

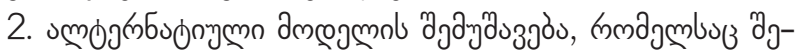

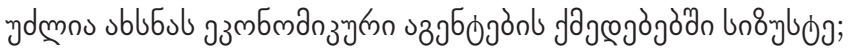

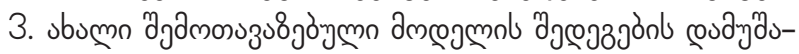

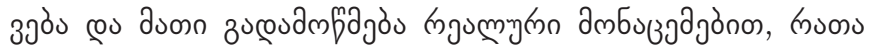

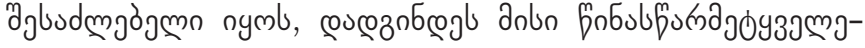

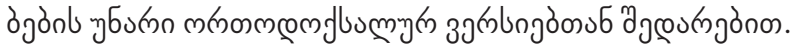

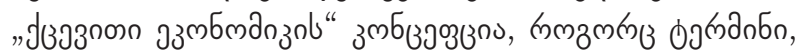

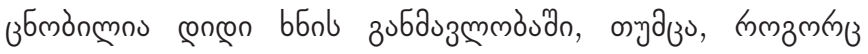

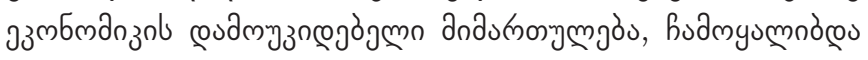




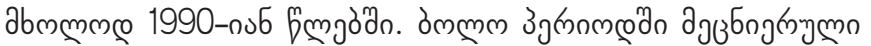

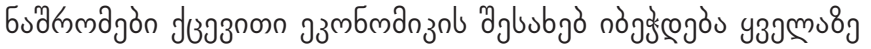

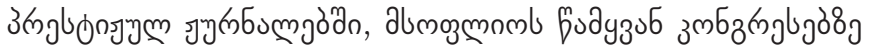

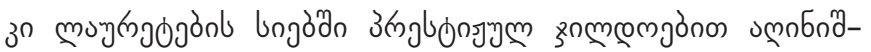

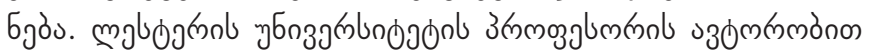

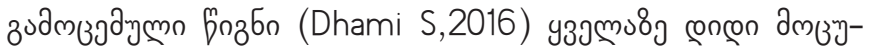

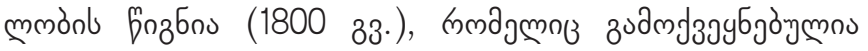
Oxford University Press-nl angm.

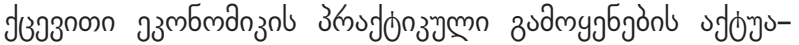

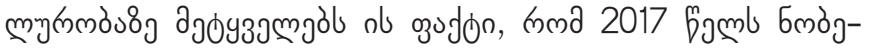

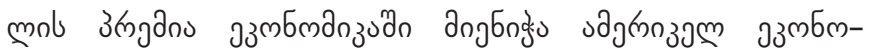

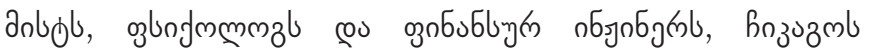

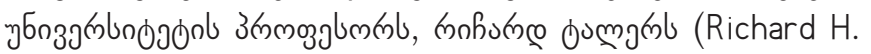

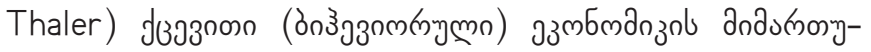

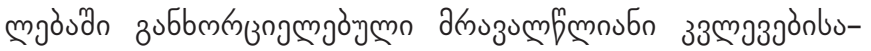

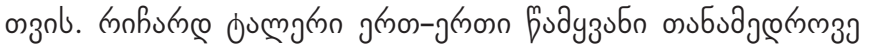

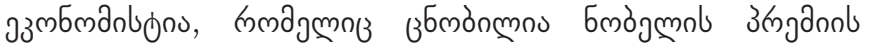

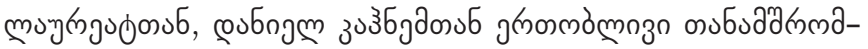

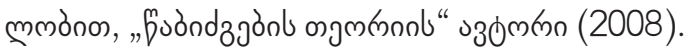

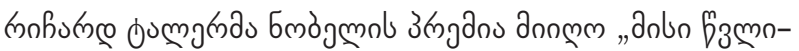

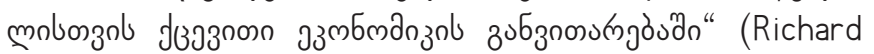
Thaler, Prize Lecture, 2017).

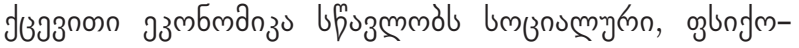

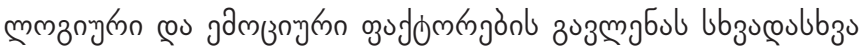

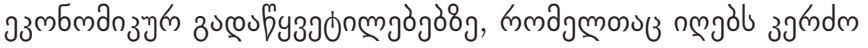

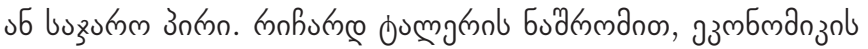

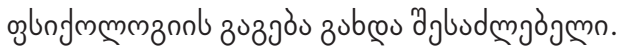

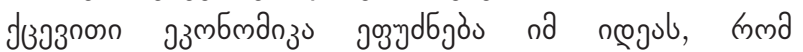

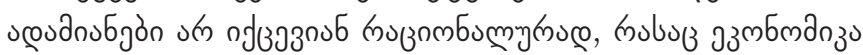

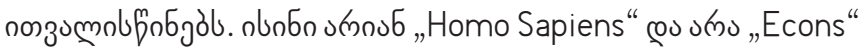

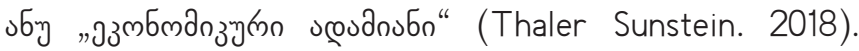

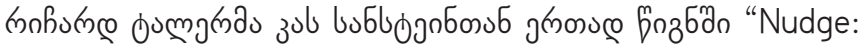
Improving Decisions About Healths, Wealth and Happiness"

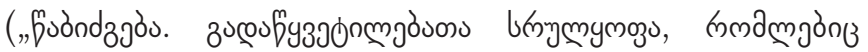

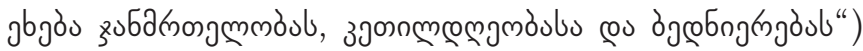

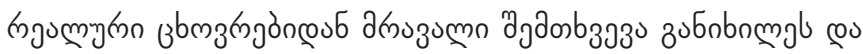

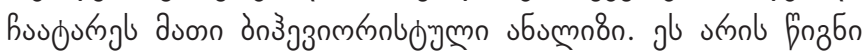

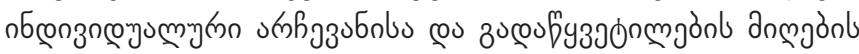

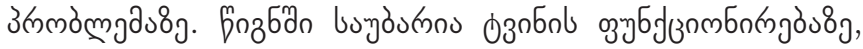

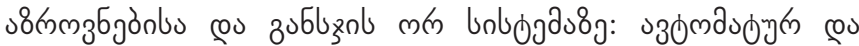
mлogm H. Thaler, 2008).

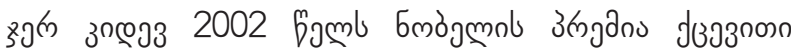

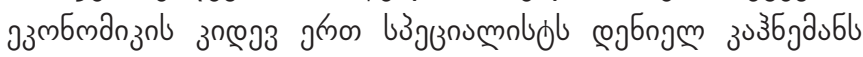

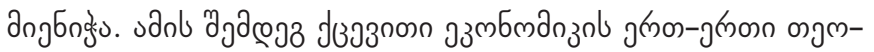

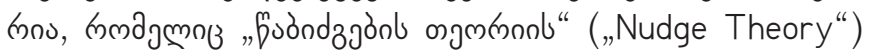

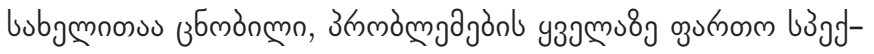

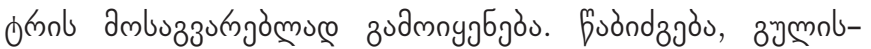

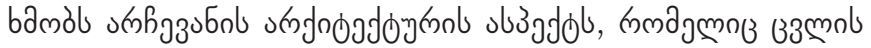

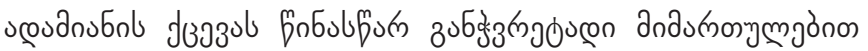

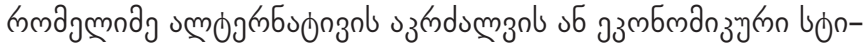

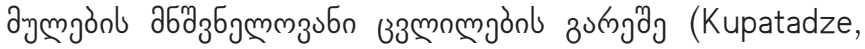
2018).

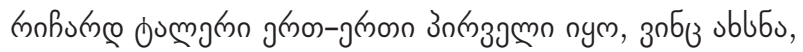

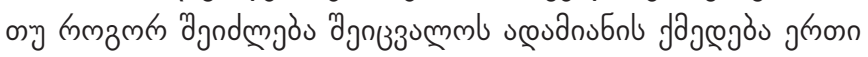

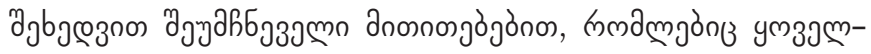

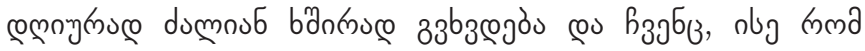

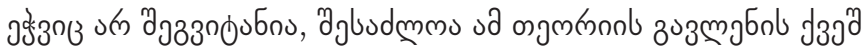

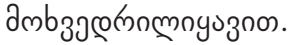

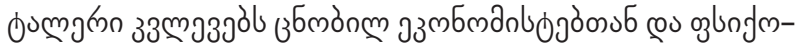

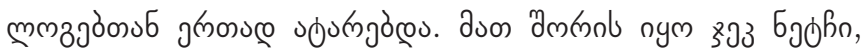

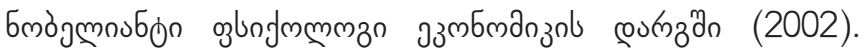

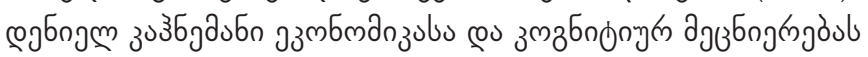

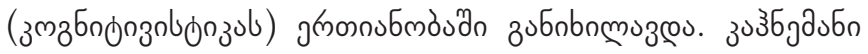

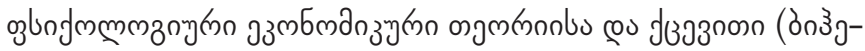

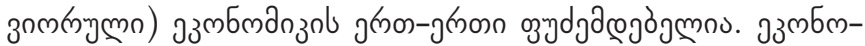

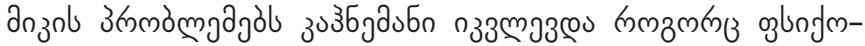

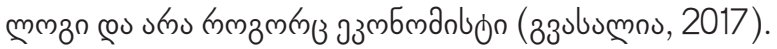

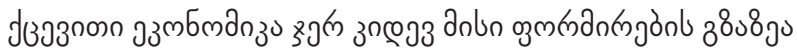

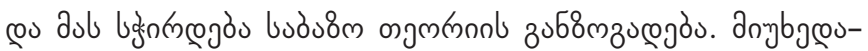

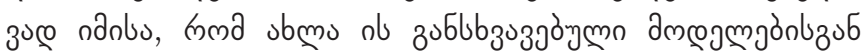

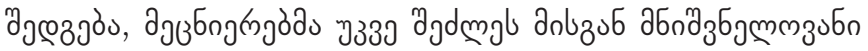

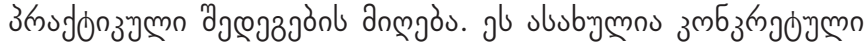

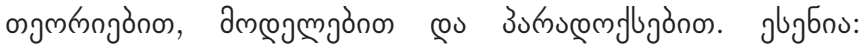

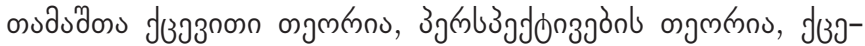

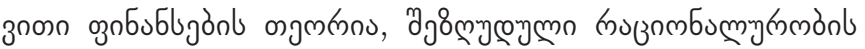

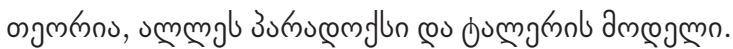

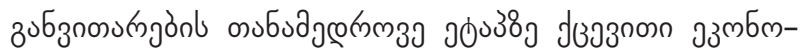

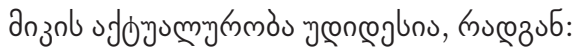

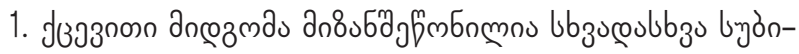

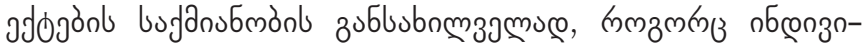

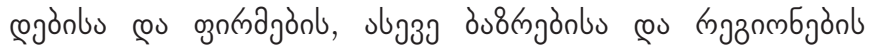
aurnoszol ubzucoulbzu combjö;

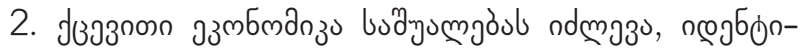

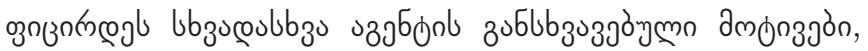

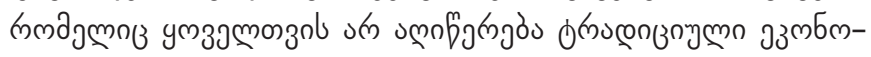

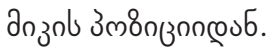

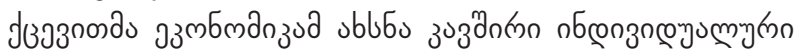

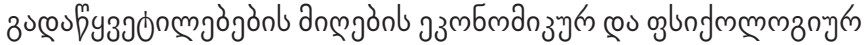

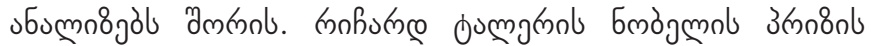

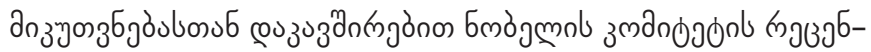

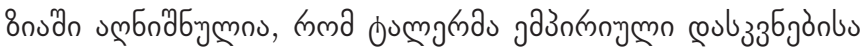

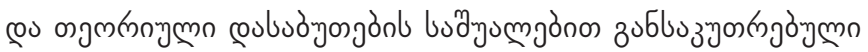

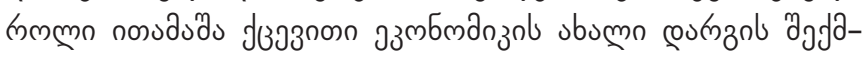

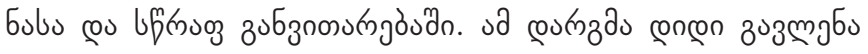
amubon cos उmmnon zu०o.

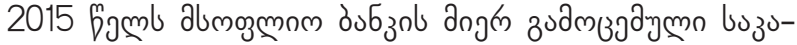

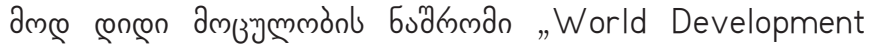
Report 2015: Mind, Society, and Behavior" ufpmprọ 


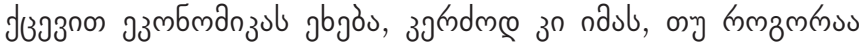

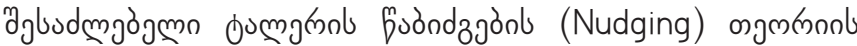

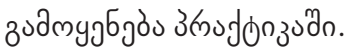

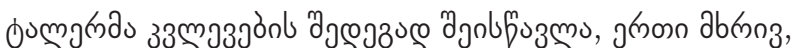

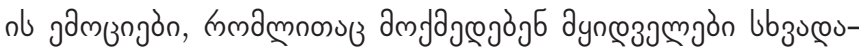

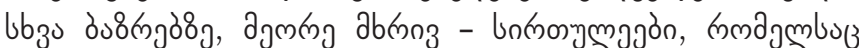

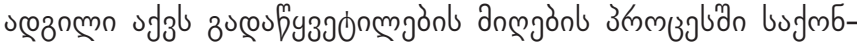

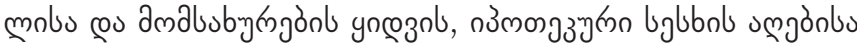

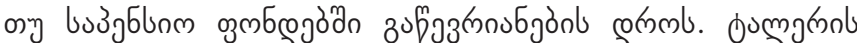

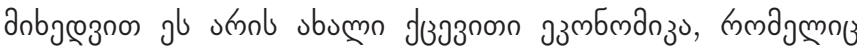

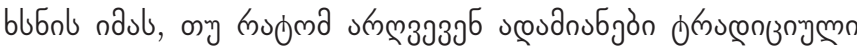

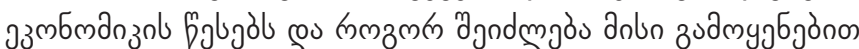

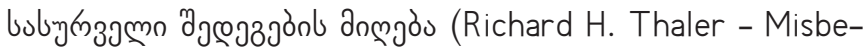
having. The Making of Behavioral Economics, 2015).

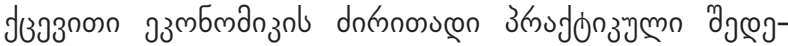

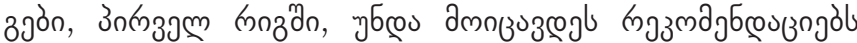

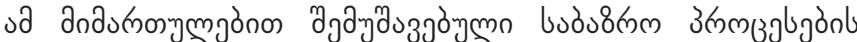

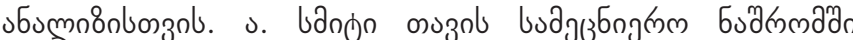

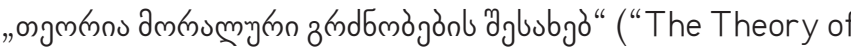

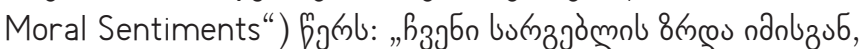

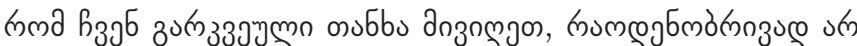

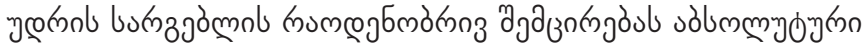

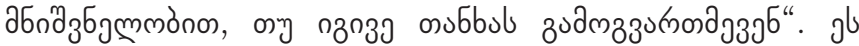

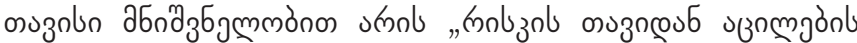
змб(зазъзиј" - Loss Aversion (Smith, 1759).

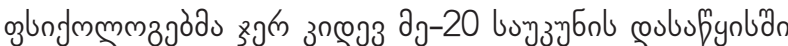

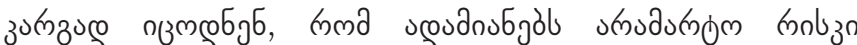

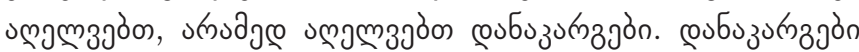

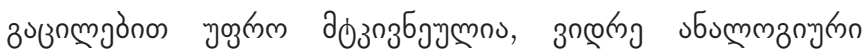

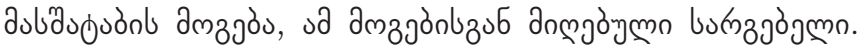

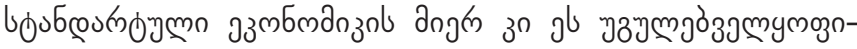

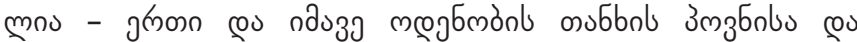

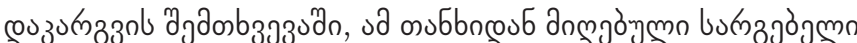

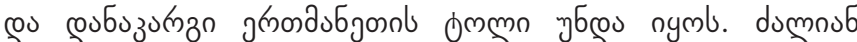

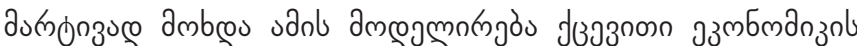

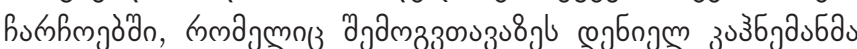

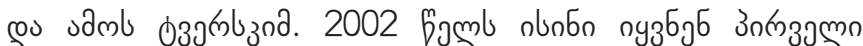

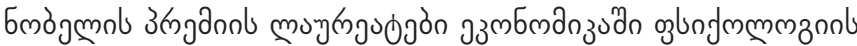

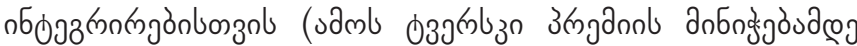

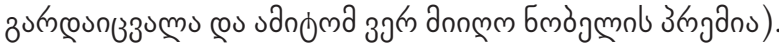

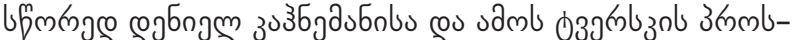

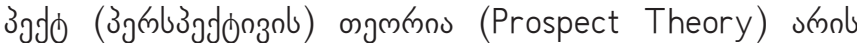

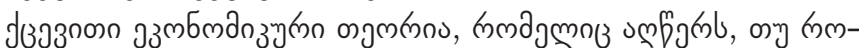
змк мз з

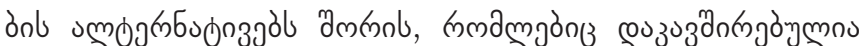

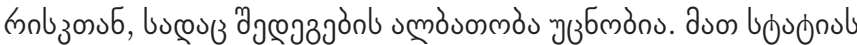

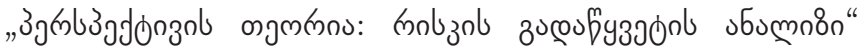

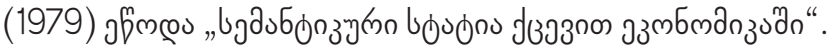

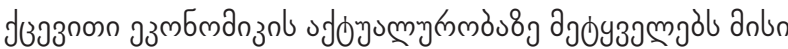

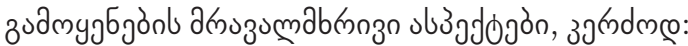

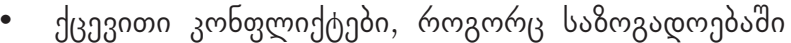

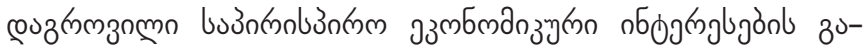

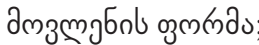

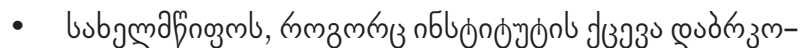

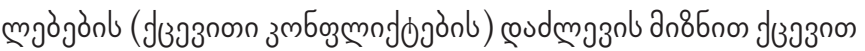
ззмбмдаз зодо;

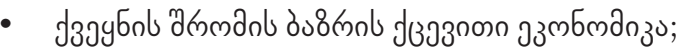

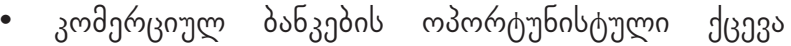

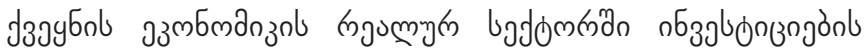

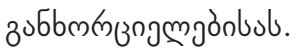

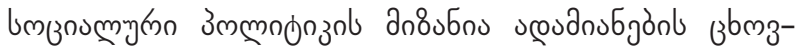

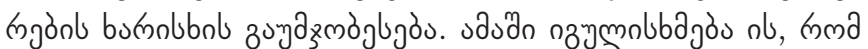

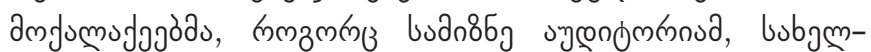

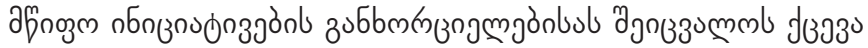

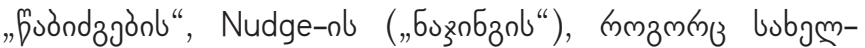

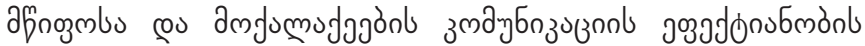

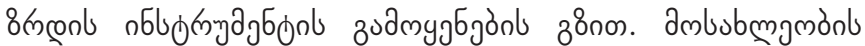

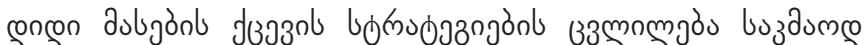

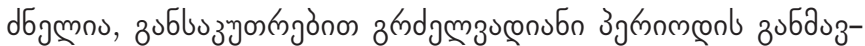

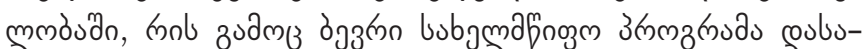

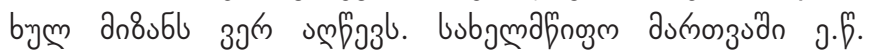

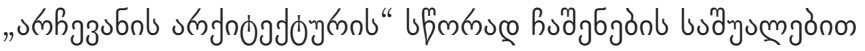

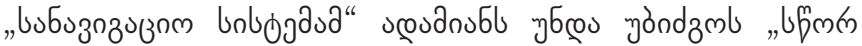

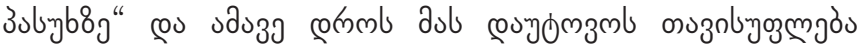
उзgmmb n

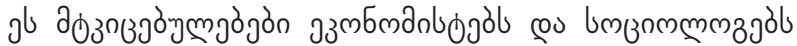

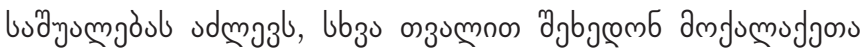

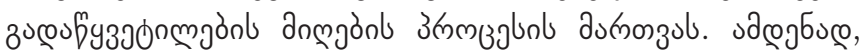

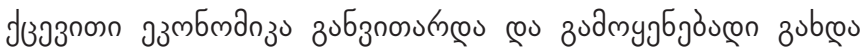

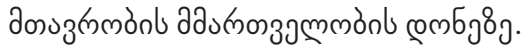

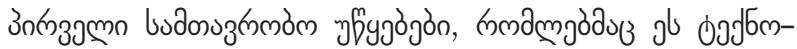

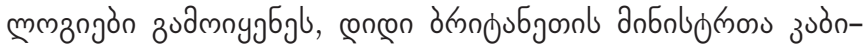

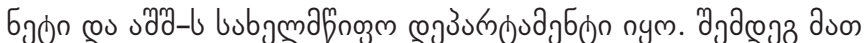

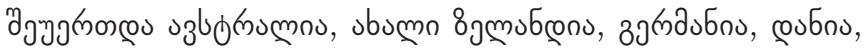
buognutägon.

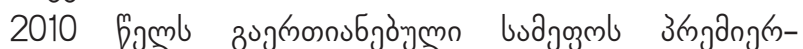

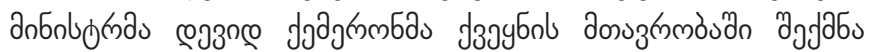

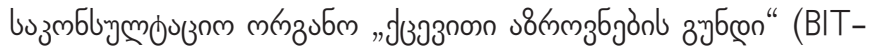

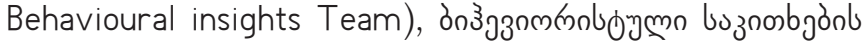

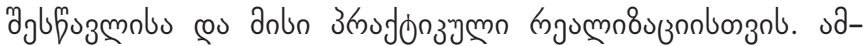

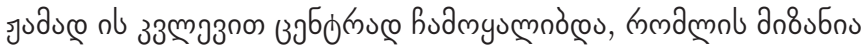

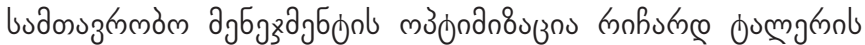

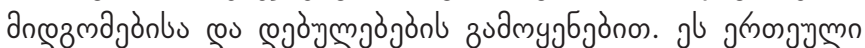

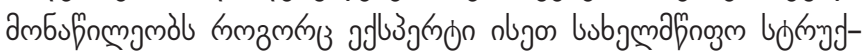

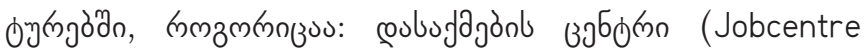

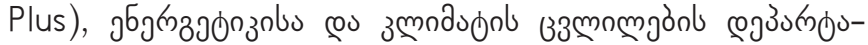
ajбon (Department for Energy and Climate Change),

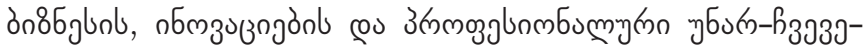

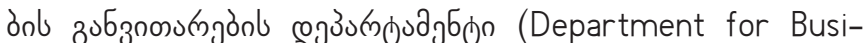
ness, Innovation and Skills), ejoufumugnol 3mmozonls 


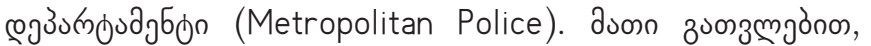

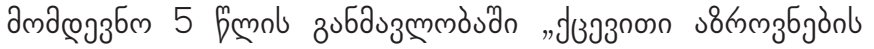

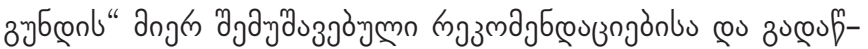

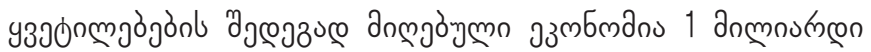

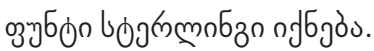

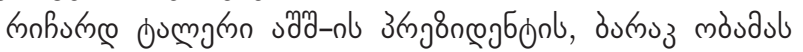

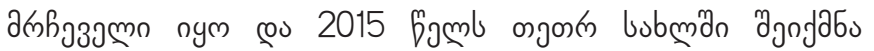

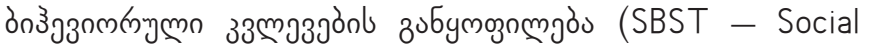

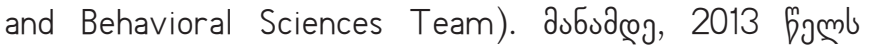

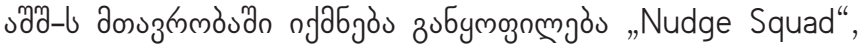

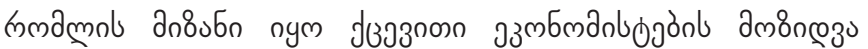

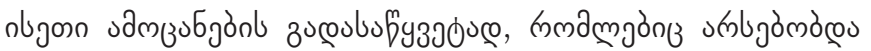

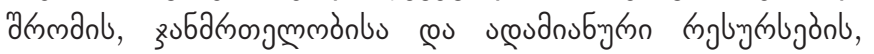

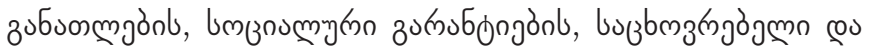

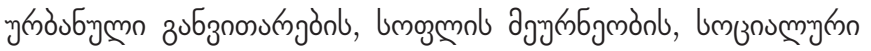

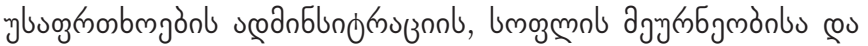

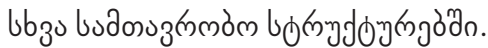

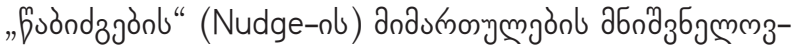

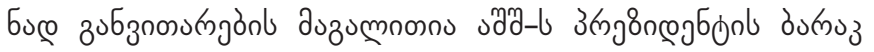

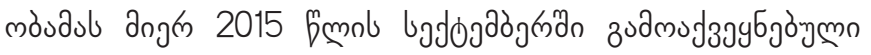

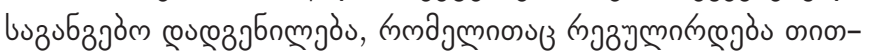

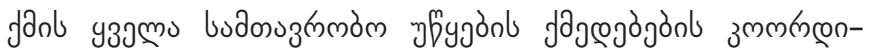
Esżnu J

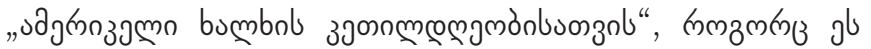

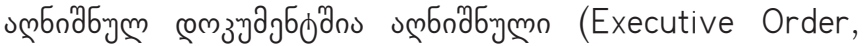
Using Behavioral Science Insights to Better Serve the American People. The White House. September 15, 2015).

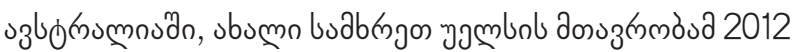

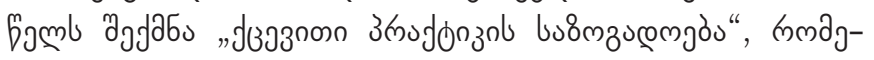

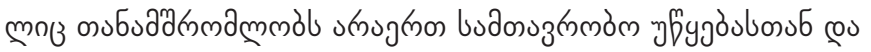

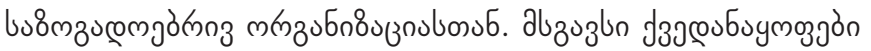

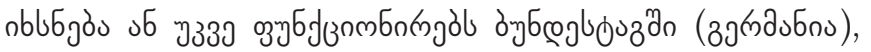

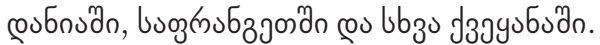

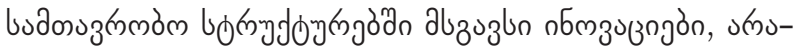

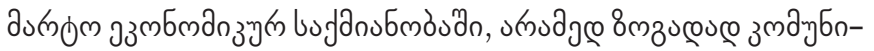

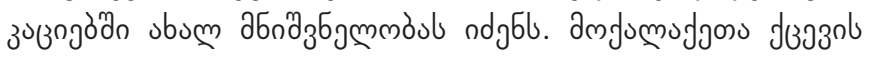

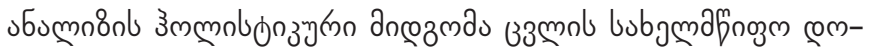

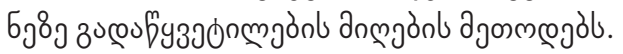

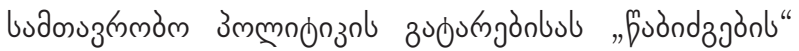

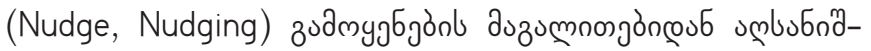

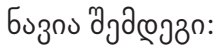

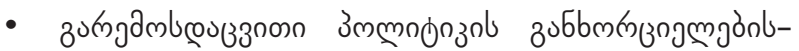

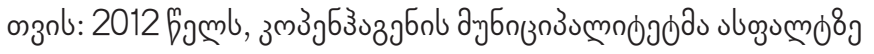

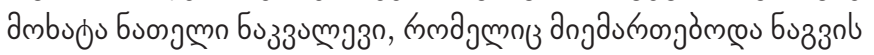

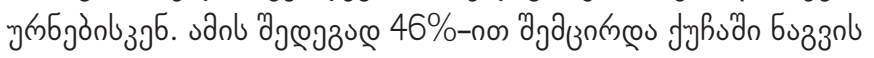
cougno (Holland, R. W., Hendriks, M., \& Aarts, H. 2005);

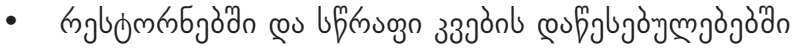

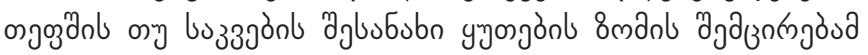

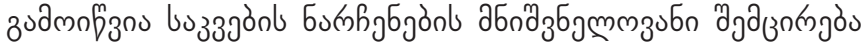
(Kallbekken, S., \& Sælen, 2013).

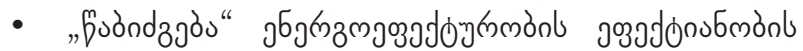

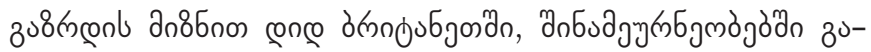

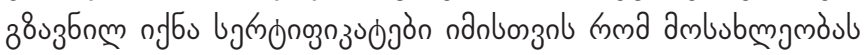

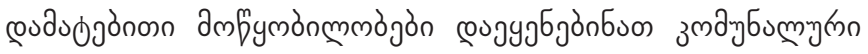

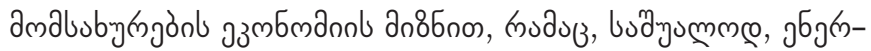

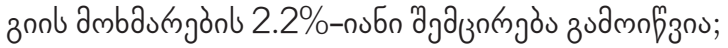

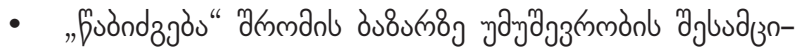

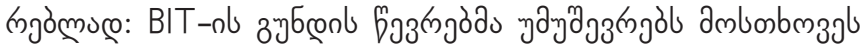

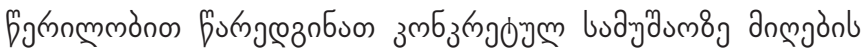

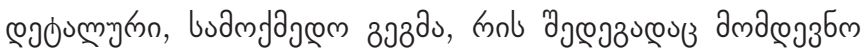

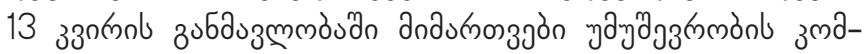

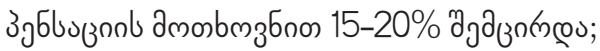

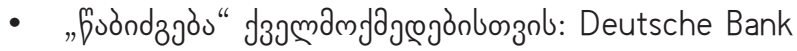

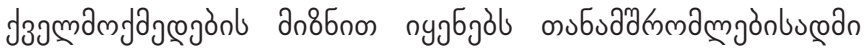

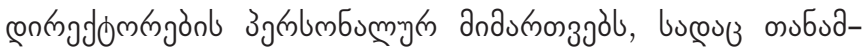

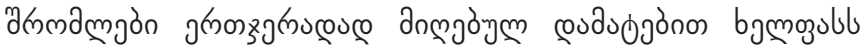

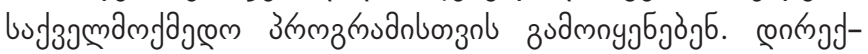

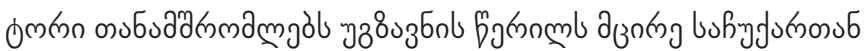

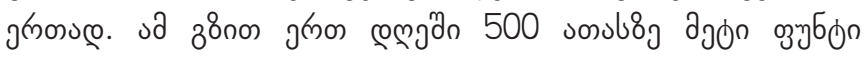

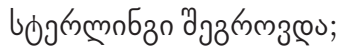

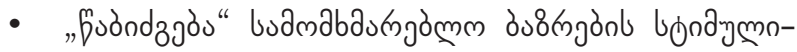

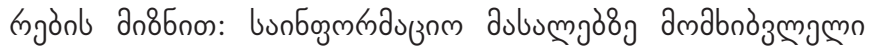

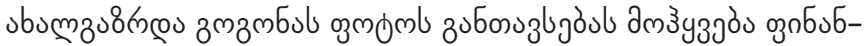

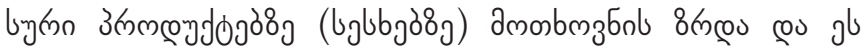

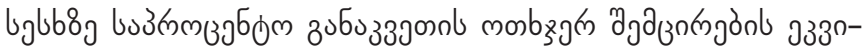

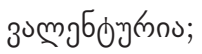

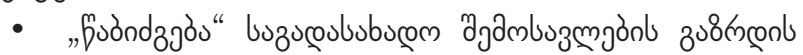

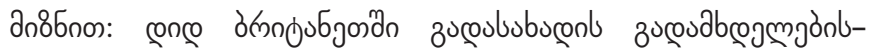

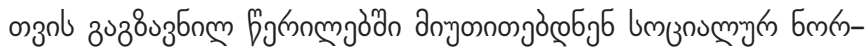

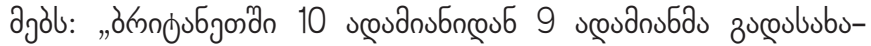

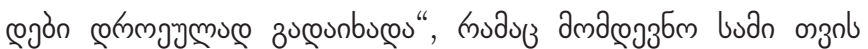

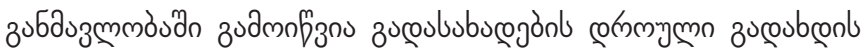

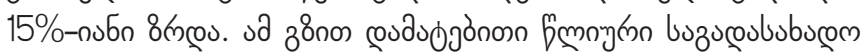

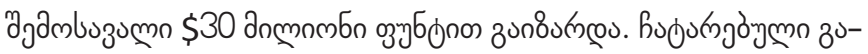

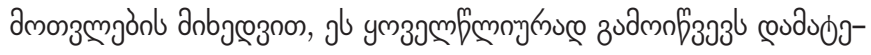

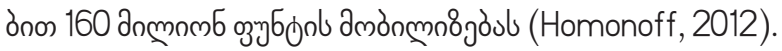

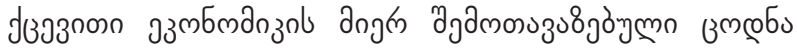

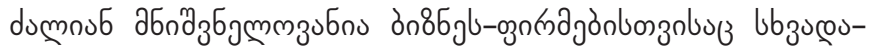

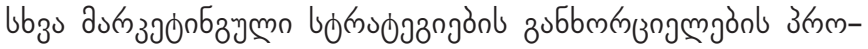

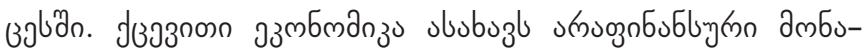

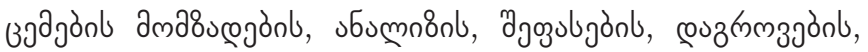

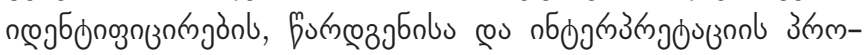

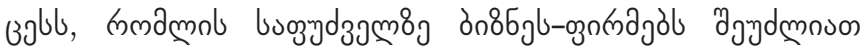

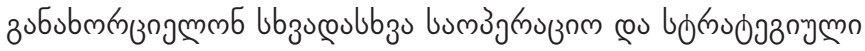

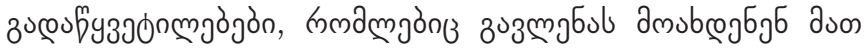

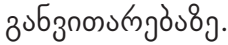




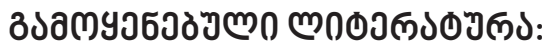

1. Colin Camerer and George Loewenstein, "Behavioral Economics: Past, Present, Future," Chapter 1 in "Advances"; manuscript, http://www.hss.caltech.edu/ camerer/ribe239.pdf

2. Daniel Kahneman, "Maps of Bounded Rationality: Psychology for Behavioral Economics,"American Economic Review 93 (2003), http://links.jstor.org/sici?sici=0002-8282\%28200312\%2993\%3A5\%3C1449\%3AMOBRPF\%3E2.0.CO\% 3B2-\%23

3. Dhami S.(2016). TheFoundations of Behavioral Economic Analysis.Oxford:OxfordUniversityPress. https://www. researchgate.net/publication/321190977_Sanjit_Dhami_The_Foundations_of_Behavioral_Economic_Analysis

4. Kallbekken, S., \& Sælen, H. (2013). „Nudging“ hotel guests to reduce food waste as a win-win environmental measure. Economics Letters. https://www.researchgate.net/publication/256994864_>Nudging>_hotel_guests_to_reduce_ food_waste_as_a_win-win_environmental_measure

5. Richard H. Thaler, Cass R. Sunstein-Nudge: Improving Decisions About Health, Wealth, and Happiness». Yale University Press, 2008 https://www.researchgate.net/file.PostFileLoader.html?id=53abe564cf57d7df1e8b45f4\&assetKey=AS \%3A273548994646025\%401442230571326

6. Richard H. Thaler, Prize Lecture, From Cashews to Nudges: The Evolution of Behavioral Economics, 2017 https:// www.nobelprize.org/prizes/economic-sciences/2017/thaler/lecture/

7. Richard H. Thaler - Misbehaving. The Making of Behavioral Economics, 2015.

8. The Nudge blog, Where is behavioral economics headed in the world of marketing? October 9, $2011 \mathrm{http}: / /$ nudges. org/2011/10/09/where-is-behavioral-economics-headed-in-the-marketing-worlding/

9. Holland, R. W., Hendriks, M., \&Aarts, H. (2005). Smells Like Clean Spirit Nonconscious Effects of Scent on Cognition and Behavior. Psychological Science, 16(9), 689-693. http://goallab.nl/publications/documents/Holland,\%20Hendriks, \%20 Aarts\%20(2005)\%20-\%20noncsious\%20effects\%20of\%20scent\%20on\%20behavior.pdf

10. Homonoff, T. A. (2012). Can Small Incentives Have Large Effects? The Impact of Taxes versus Bonuses on Disposable Bag Use. https://wagner.nyu.edu/files/faculty/publications/Homonoff\%20-\%20Can\%20Small\%20Incentives\%20 Have\%20Large\%20Effects_0.pdf

11. Executive Order - Using Behavioral Science Insights to Better Serve the American People. The White House. September 15, 2015. https://www. whitehouse.gov/the-press-office/2015/09/15/executive-order-using-behavioralscience-insights-better-serve-american

12. 12. Smith, A. (1759). Theory of Moral Sentiments [adam smit, teorianravstvennix chuvst], e-reading.club, (In Russion). https://www.e-reading.club/book.php?book=1039709

13. Kupatadze, G. (2018). From the architecture of choice to the Nobel Laureate [archevanis arqiteqturidan nobelis laureatobamde] (In Georgian). https://1tv.ge/analytics/archevanis-arqiteqturidan-nobelis-laureatobamde/

14. Gvasalia, G. (2017). The Behavioral Economic Theory of Richard Thaler. [richard seileris bihevioristuli ekonomikis teoria] Journal: Audit, accounting, finance. N11 (215), (In Georgian).

15. https://www.nobelprize.org/prizes/economic-sciences/2017/thaler/lecture/

16. World Development Report 2015: Mind, Society, and Behavior, By World Bank https://books.google.ge/books/ about/World_Development_Report_2015.html?id=bjP5BgAAQBAJ\&printsec=frontcover\&source=kp_read_ button\&redir_esc=y№v=onepage\& $q \& f=f a l s e$

17. https://www.bi.team/

18. https://sbst.gov/ 


\title{
THEORETICAL BASICS AND PRACTICAL ASPECTS OF BEHAVIORAL ECONOMICS
}

\author{
MARINE NATSVALADZE \\ Associate Professor, Doctor of Economics \\ Ivane Javakhishvili Tbilisi State University, Georgia \\ marine.natsvaladze@tsu.ge
}

KEYWORDS: BEHAVIORAL ECONOMICS, ECONOMIC DECISION MAKING, ECONOMICS AND PSYCHOLOGY, ECONOMIC POLICY.

For citation: Natsvaladze, M. (2019). Theoretical Foundations and Practical Aspects of Behavioral Economics, Globalization And Business, №8, pp. 68-73. https://doi.org/10.35945/gb.2019.08.007

\section{SUMMARY}

Traditional Economics looks at the persons as at some kind of rational machine which takes into consideration all available information and then makes optimal decision. Reality is rather different. The behavioral economics claims that there is no rational "economic human" and probably will never exist. Person's behavior is irrational and this irrationality is not random and clueless. Vice-versa - this irrationality is systemic and predictable.

Behavioral economics explores what affects people's economic decisions and the consequences of those decisions for market prices, returns, and resource allocation. Traditional economic research assumes that people's economic decisions are based on the rule of maximizing utility.

Behavioral economics uses experiments that observe human behavior in order to uncover how we think. Behavioral economics has been called the science of decision-making. It is a growing academic discipline which uses experiments that observe human behavior in order to uncover how we think. Behavioral economics is about understanding common decision mistakes that people make and why they make them. In particular, a large aspect of behavioral economics is concerned with the gap between intention and action.

Classical economic theory assumes that individuals are rational. However, in the real world, we often see irrational behavior - decisions which donst maximize utility but can cause a loss of economic welfare. It means economists need to take into account the potential for irrationality.

Successful marketers must have a profound understanding of the consumer's thought process in order to create a successful marketing campaign. By understanding the consumer's decision-making process, marketers are able to develop value propositions that really fit the consumer's needs. The importance of understanding behavioral economics for marketers is immeasurable as it allows for a better understanding of the human mind. Behavioral economics allows marketing professionals to optimize marketing strategies and get real results.

In the article are reviewed applied aspects of behavioral economics, also theoretical and practical results of researches. These results will be useful in company management, for politicians, in private decision making as they give different perspective to rational-functional models. In case of ignoring the interdisciplinary approaches, integration of economics and psychology can result in waste of resources and wrong decisions. 